\title{
EXTENDING A MEASURE FROM A RING TO A SIGMA-RING
}

\author{
ROY A. JOHNSON
}

\begin{abstract}
For each measure on a ring, there is a smallest measure on the generated sigma-ring such that ring members of finite measure keep the same measure. Conditions are given under which this smallest semi-extension preserves sums and order.
\end{abstract}

1. Introduction. A (nonnegative, countably additive) measure $\mu$ on a ring $\mathcal{R}$ is said to be semifinite if

$$
\mu(E)=\sup \{\mu(E \cap P): P \in R \text { and } \mu(P)<\infty\}
$$

for all $E$ in $R$ [1, Exercise 25.9]. Although $\mu$ can be extended by means of outer measure to a measure $\mu^{L}$ on the smallest sigma-ring $\mathcal{S}$ containing $R$, this largest extension $\mu^{L}$ may fail to be semifinite even if $\mu$ is semifinite. However, a semifinite measure $\mu$ on $\Re$ has a smallest extension to a measure $\mu^{s}$ on $\mathcal{S}[4$, p. 757], and this smallest extension $\mu^{s}$ is indeed semifinite [4, p. 758]. Luther has shown that semifiniteness of the largest extension $\mu^{L}$ implies the equality of $\mu^{S}$ and $\mu^{L}$, so that a semifinite measure $\mu$ on $\Re$ has a unique extension to a measure on $\mathcal{S}$ whenever $\mu^{L}$ is semifinite [4, Theorem 3].

Now let $\mu$ and $\nu$ be measures on $\mathcal{R}$ having unique extensions to corresponding measures $\mu^{\#}$ and $\nu^{\#}$ on $\mathcal{S}$. It can be seen that their sum has a unique extension $(\mu+\nu)^{\#}$ and that $(\mu+\nu)^{\#}=\mu^{\#}+\nu^{\#}$. Moreover, Huang has shown in effect that the relationship $\mu<\nu$ on $\Re$ implies the relationship $\mu^{\#}<\nu^{\#}$ on $\mathcal{S}$ in this case [3, Theorem 2].

Whereas Luther [4] and Huang [3] are primarily interested in the case where extension is unique, the focus of this paper is on additivity and monotonicity for the case in which measures have more than one extension. In particular, if $\mu$ and $\nu$ are (not necessarily semifinite) measures on $R$, then it is easy to see that $(\mu+\nu)^{L}=\mu^{L}+\nu^{L}$ and that the relationship $\mu<\nu$ implies $\mu^{L}<\nu^{L}$. On the other hand, even if $\mu$ and $\nu$ are semifinite, the corresponding statements for $\mu^{s}$ and $\nu^{s}$ can be false. For example, let $X$ be the real line and let $R$ be the ring of sets which are finite or cofinite in $X$. If $E \in \Re$, let $\mu(E)=0$ if $E$ is finite and let $\mu(E)=1$ if $E$ is cofinite. If $E \in \Re$, let $\nu(E)$ be the number of positive integers in $E$. Now if $A$ is the complement in $X$ of the set of positive integers, then $(\mu+\nu)^{S}(A)=0$ whereas $\mu^{s}(A)=1$ and $\nu^{s}(A)=0$ (cf. [5, Exercise 2.3.20]). Notice, moreover, that the statement $\mu^{S}<\nu^{S}$ is false even though $\mu<\nu$.

Received by the editors April 2, 1979.

AMS (MOS) subject classifications (1970). Primary 28A05, 28A10. 
In Theorems 3.1 and 3.2, necessary and sufficient conditions are given so that $(\mu+\nu)^{s}=\mu^{s}+\nu^{s}$ or so that $\mu^{s}<\nu^{s}$.

The author wishes to thank the referee for suggestions which have improved the exposition of this paper.

2. Extensions and semi-extensions of a measure. This section consists mainly of the details used in "extending" a nonsemifinite measure on the ring $R$ to a measure on the generated sigma-ring $\mathcal{S}$. Suppose $\mu$ is a measure on $R$. Following $\left[4\right.$, p. 757], the measure $\mu^{\#}$ on $\mathcal{S}$ is an extension (semi-extension) of $\mu$ if $\mu^{\#}(E)=$ $\mu(E)$ for all $E$ in $\Re$ (for all $E$ in $\Re$ such that $\mu(E)$ is finite). Since all semi-extensions of $\mu$ agree on $\Re_{\varphi}$, the class of sets in $\Re$ having finite $\mu$-measure, it follows that they agree on $\delta_{\varphi}$, the class of sets in $\delta$ contained in such ring members. Hence, all semi-extensions of $\mu$ agree on $\mathcal{S}_{\varphi_{0}}$, the class of sets in $\mathcal{S}$ which can be covered by countably many members of $\delta_{\varphi}$.

As is well known, $\mu$ has an extension $\mu^{L}$ defined by the formula

$$
\mu^{L}(F)=\inf \left\{\sum \mu\left(E_{i}\right): \text { each } E_{i} \in \Re \text { and } F \subset \cup E_{i}\right\}
$$

for all $F$ in $\delta\left[2\right.$, Theorems 10.A and 11.B]. Moreover, $\mu$ has a semi-extension $\mu^{s}$ defined by the formula

$$
\mu^{s}(F)=\sup \left\{\mu^{L}(F \cap P): P \in \Re \text { and } \mu(P)<\infty\right\}
$$

for all $F$ in $\mathcal{S}\left[4\right.$, p. 757]. If $\mu^{\#}$ on $\mathcal{S}$ is any semi-extension of $\mu$, then $\mu^{S}<\mu^{\#}<$ $\mu^{L}$ [4, Theorem 1(ii)]. Conversely, if $\nu^{\#}$ is a measure on $\mathcal{S}$ which lies between $\mu^{S}$ and $\mu^{L}$, then $\nu^{\#}$ agrees with $\mu$ on $\Re_{\varphi}$ so that $\nu^{\#}$ is a semi-extension of $\mu$. If $\mu^{\#}$ is a semi-extension of $\mu$, then $\mu^{S}$ and $\mu^{L}$ can be described in terms of $\mu^{\#}$ as follows. For each $F$ in $\delta$, the value $\mu^{S}(F)$ is given by $\sup \left\{\mu^{\#}(F \cap M): M \in \mathcal{\delta}_{\varphi}\right\}$, and $\mu^{L}(F)$ is given by $\mu^{\#}(F)$ if $F \in \mathcal{S}_{\varphi 0}$ and $\infty$ otherwise. Hence, if $\mu^{L}(F)<\infty$, then all semi-extensions of $\mu$ agree on $F$. This fact will be used in the proof of Theorem 3.1 .

For each measure $\mu$ on a ring, there is a corresponding semifinite measure $\mu_{s}$ defined on that ring by the formula $\mu_{s}(F)=\sup \{\mu(F \cap P): \mu(P)<\infty\}$ for each ring member $F\left[1\right.$, Theorem 10.1]. Notice that $\mu_{s}$ is the smallest measure agreeing with $\mu$ on sets of finite $\mu$-measure. Hence, $\mu=\mu_{s}$ if and only if $\mu$ is semifinite. The measure $\mu_{s}$ is of interest to us for two reasons. First, $\mu^{s}=\left(\mu^{L}\right)_{s}$. That is, $\mu^{s}$ is simply the smallest measure on $\mathcal{S}$ which agrees with $\mu^{L}$ on sets of finite $\mu^{L}$-measure. Secondly, $\mu^{S}=\left(\mu_{s}\right)^{S}$ so that the semi-extension $\mu^{s}$ is actually an extension of $\mu_{s}$. In other words, the function sending $\mu$ into $\mu^{s}$ does not care what $\mu$ looks like; instead it seeks out and acts on $\mu_{s}$. As a consequence, every semi-extension of $\mu$ is an extension of $\mu_{s}$ and conversely (cf. [4, Theorem 2]).

An advantage of semi-extensions over extensions is that a measure $\mu$ on $R$ always has a smallest semi-extension to a measure on $\mathcal{S}$, but $\mu$ need not have a smallest extension to a measure on $\mathcal{S}$. For example, let $R$ be the ring of subsets of the real line generated by sets of the form $[a, b)$ and let $\delta$ be the Borel sets of the real line. Let $\mu$ be the measure on $\Re$ which is $\infty$ for all nonempty sets and 0 for the 
empty set. If $F \in \mathcal{S}$, let $\mu^{\#}(F)\left(\mu^{\# \#}(F)\right)$ be the number of rational (resp. irrational) numbers in $F$. Then $\mu^{\#}$ and $\mu^{\# \#}$ agree with $\mu$ for all sets in $\mathcal{R}$ and their infimum is the zero measure on $\mathcal{S}$. Hence, there is no smallest extension of $\mu$ to a measure on $\mathfrak{S}$.

3. Effect of smallest semi-extensions on sums and order. In this section, necessary and sufficient conditions are given so that $(\mu+\nu)^{s}=\mu^{s}+\nu^{s}$ or so that $\mu^{s}<\nu^{s}$. If $\mu^{\#}$ and $\nu^{\#}$ are measures on $\mathcal{S}$, we say that $\nu^{\#}$ is absolutely continuous with respect to $\mu^{\#}$, denoted $\nu^{\#} \ll \mu^{\#}$, if $\nu^{\#}(F)=0$ whenever $F \in \mathcal{S}$ and $\mu^{\#}(F)=0$ $[2$, p. 124]. We will not need the notion of absolute continuity for measures on the ring $R$.

THEOREM 3.1. Suppose $\mu$ and $\nu$ are measures on the ring $R$. Then

(1) $(\mu+\nu)^{s}<\mu^{s}+\nu^{s}$.

(2) $(\mu+\nu)^{S}=\mu^{S}+\nu^{s}$ if and only if $\mu^{S}+\nu^{S} \ll(\mu+\nu)^{S}$.

Proof. (1) Suppose $F \in \mathcal{S}$ and suppose $(\mu+\nu)^{S}(F)>k$. We show that $\mu^{S}(F)$ $+\nu^{S}(F)>k$. Choose $G$ in $R$ such that $(\mu+\nu)(G)<\infty$ and such that $(\mu+\nu)^{S}(F \cap G)>k$. Let $H=F \cap G$. Since $(\mu+\nu)^{L}(H)$ is finite, it follows that all semi-extensions of $\mu+\nu$ agree on $H$. In particular, $(\mu+\nu)^{S}(H)=\mu^{S}(H)+$ $\nu^{s}(H)$. Hence,

$$
k<(\mu+\nu)^{S}(H)=\mu^{S}(H)+\nu^{S}(H)<\mu^{S}(F)+\nu^{S}(F) .
$$

(2) In view of (1), it suffices to show that $\mu^{S}(F)+\nu^{s}(F)=(\mu+\nu)^{S}(F)$ whenever $(\mu+\nu)^{S}(F)$ is finite. Suppose, then, that $F \in \mathcal{S}$ and that $(\mu+\nu)^{S}(F)<$ $\infty$. By the definition of $(\mu+\nu)^{S}(F)$, we may choose a sequence of sets $\left\{G_{n}\right\}$ in $R$ such that $(\mu+\nu)\left(G_{n}\right)<\infty$ for each $n$ and such that

$$
(\mu+\nu)^{S}(F)=\sup \left\{(\mu+\nu)^{S}\left(F \cap G_{n}\right)\right\} .
$$

Let $H=\cup G_{n}$. Then $(\mu+\nu)^{S}(F-H)=0$, so that $\mu^{S}(F-H)+\nu^{S}(F-H)=$ 0 . Since $F \cap H$ is a countable union of sets in $\delta$ having finite $(\mu+\nu)^{L}$-measure, it follows that all semi-extensions of $\mu+\nu$ agree on $F \cap H$. In particular, $\mu^{s}(F \cap H)+\nu^{s}(F \cap H)=(\mu+\nu)^{s}(F \cap H)$. Hence,

$$
\begin{aligned}
\mu^{S}(F)+\nu^{S}(F) & =\mu^{S}(F \cap H)+\nu^{S}(F \cap H) \\
& =(\mu+\nu)^{S}(F \cap H)=(\mu+\nu)^{s}(F) .
\end{aligned}
$$

If $\mu<\nu$, then there is a smallest measure $\pi$ on $\Re$ such that $\mu+\pi=\nu$ (cf. [6, Exercise 11.5d]). Simply let

$$
\pi(E)=\sup \{\nu(E \cap A)-\mu(E \cap A): A \in \Re \text { and } \mu(E \cap A)<\infty\}
$$

for each $E$ in $\Re$. In the following Lemma we shall use the fact that if $\pi(E)<\infty$, then $\mu(E \cap A)<\infty$ for precisely those sets $A$ in $\Re$ for which $\nu(E \cap A)<\infty$.

LEMMA. Suppose $\mu$ and $\nu$ are measures on $R$ such that $\left(\mu_{s}\right)^{L} \ll \nu^{L}$ (for example, $\mu_{s}<\nu$ or $\left.\mu<\nu\right)$ and such that if $\mu(E)$ is finite, then

$$
\mu(E)=\sup \{\mu(E \cap A): A \in \Re \text { and } \nu(E \cap A)<\infty\} \text {. }
$$


Then $\mu^{s} \ll \nu^{s}$. If $\mu<\nu$ and $\pi$ is the smallest measure on $R$ such that $\mu+\pi=\nu$, then $\pi^{s} \ll \nu^{s}$.

Proof. Suppose $\mu^{S}(F)>0$. Then by the definition of $\mu^{S}(F)$, there exists $E$ in $\mathscr{R}$ such that $\mu(E)<\infty$ and such that $\mu^{L}(F \cap E)>0$. Hence, $\mu^{L}(E-F)<\mu(E)$. By hypothesis, we may choose $A$ in $\mathscr{R}$ such that $\nu(E \cap A)<\infty$ and such that $\mu(E \cap A)>\mu^{L}(E-F)$. Necessarily, $\mu^{L}(F \cap E \cap A)>0$. Since

$$
\left(\mu_{s}\right)^{L}(F \cap E \cap A)=\mu^{L}(F \cap E \cap A)
$$

and since $\left(\mu_{s}\right)^{L} \ll \nu^{L}$ by hypothesis, we have $\nu^{L}(F \cap E \cap A)>0$. Then since $\nu^{L}(F \cap E \cap A)$ is finite, we have $0<\nu^{s}(F \cap E \cap A)<\nu^{s}(F)$. Hence, $\mu^{s} \ll \nu^{s}$.

Now suppose $\mu<\nu$ and suppose $\pi$ is the smallest measure on $\mathscr{R}$ such that $\mu+\pi=\nu$. If $\pi(E)<\infty$, then

$$
\begin{aligned}
\pi(E) & =\sup \{\nu(E \cap A)-\mu(E \cap A): A \in \Re \text { and } \mu(E \cap A)<\infty\} \\
& =\sup \{\nu(E \cap A)-\mu(E \cap A): A \in \Re \text { and } \nu(E \cap A)<\infty\} \\
& =\sup \{\pi(E \cap A): A \in \Re \text { and } \nu(E \cap A)<\infty\} .
\end{aligned}
$$

Clearly, $\pi<\nu$, so that $\pi^{s} \ll \nu^{s}$ by the first half of the Lemma.

THEOREM 3.2. Suppose $\mu$ and $\nu$ are measures on the ring $\mathscr{R}$. Then $\mu^{s}<\nu^{s}$ if and only if $\mu_{s}<\nu$ and $\mu^{s} \ll \nu^{s}$. Hence, if $\mu_{s}<\nu$ and if

$$
\mu(E)=\sup \{\mu(E \cap A): A \in \Re \text { and } \nu(E \cap A)<\infty\}
$$

whenever $\mu(E)<\infty$, then $\mu^{S}<\nu^{S}$.

Proof. The "only if" statement is clear since the restriction of $\mu^{s}$ to $\mathscr{R}$ is $\mu_{s}$. To prove the "if" statement, let $\pi$ be the smallest measure on $R$ such that $\mu+\pi=\nu$. By the preceding Lemma, $\pi^{s} \ll \nu^{s}$ so that $\mu^{s}+\pi^{s} \ll \nu^{s}$. Applying Theorem 3.1, we see that

$$
\mu^{S}<\mu^{S}+\pi^{S}=(\mu+\pi)^{S}=\nu^{S} .
$$

\section{REFERENCES}

1. S. K. Berberian, Measure and integration, Macmillan, New York, 1965.

2. P. R. Halmos, Measure theory, Van Nostrand, New York, 1950.

3. D. -Y. Huang, A note on generalized unique extension of measures, J. Austral. Math. Soc. 14 (1972), 59-61.

4. N. Y. Luther, Unique extension and product measures, Canad. J. Math. 19 (1967), 757-763.

5. A. Mukherjea and K. Pothoven, Real and functional analysis, Plenum, New York, 1978.

6. H. L. Royden, Real analysis, Macmillan, New York, 1968.

Departngent of Mathematics, Washington State University, Pulukan, Washmoton 99164 